\title{
Millimetre continuum measurements of extragalactic radio sources
}

\section{Data from 1993-1994*}

\author{
H.-P. Reuter ${ }^{1}$, C. Kramer ${ }^{2}$, A. Sievers ${ }^{2}$, G. Paubert ${ }^{2}$, R. Moreno ${ }^{2}$, A. Greve ${ }^{3}$, S. Leon ${ }^{2}$, J.F. Panis ${ }^{2}$, \\ M. Ruiz-Moreno ${ }^{2}$, H. Ungerechts ${ }^{2}$, and W. Wild ${ }^{2}$ \\ 1 Max-Planck-Institut für Radioastronomie, Auf dem Hügel 69, 53121 Bonn, Germany \\ 2 IRAM Granada, Avenida Divina Pastora 7, Nucleo Central, 18012 Granada, Spain \\ 3 IRAM Grenoble, 300 Rue de la Piscine, 38460 St. Martin d'Hères, France
}

Received June 7; accepted June 10, 1996

\begin{abstract}
Radio flux densities are presented for 118 extragalactic radio sources monitored at 90,142 and $230 \mathrm{GHz}$ with the IRAM $30 \mathrm{~m}$ telescope during 1993-1994. For the most frequently observed sources we show light curves including $30 \mathrm{~m}$-measurements published in previous papers.
\end{abstract}

Key words: galaxies: active - BL Lacertae objects: general - quasars: general — radio continuum: general — radio continuum: galaxies

\section{Introduction}

This is the fourth paper of a series summarising the results of mm-wavelength flux density monitoring of extragalactic radio sources with the IRAM $30 \mathrm{~m}$ telescope at Pico Veleta, Spain. A first selection of a sample of pointlike radio sources well suited as pointing sources for the $30 \mathrm{~m}$ telescope was made in Paper I (Steppe et al. 1988), resulting in flux density measurements in the $1-3 \mathrm{~mm}$ range of approximately 300 extragalactic radio sources. In Paper II and III (Steppe et al. 1992, 1993) monitoring results were published for sources with flux densities $\gtrsim 1 \mathrm{Jy}$ at $87.7 \mathrm{GHz}(3.4 \mathrm{~mm})$ which are now used as standard pointing sources at the telescope. The pointing catalogue used at the telescope consists mainly of quasars and BL Lac objects; a complete list of these sources is given in Table 1 (see also Wild 1995), which is published electronically.

Send offprint requests to: H.-P. Reuter, MPIfR Bonn

* Tables 1 and 4 are available electronically at the CDS via anonymous ftp 130.79 .128 .5 or at http://cdsweb.ustrasbg.fr/Abstract.html

\section{Observations and calibration}

The observations were made during the standard pointing sessions which are performed approximately every two weeks at the $30 \mathrm{~m}$ telescope. In general, three SIS receivers were used simultaneously, namely at $87.7,142$ and $230 \mathrm{GHz}$ tuned to DSB mode. The performances of the receivers are given in Table 2 (see also Wild 1995). Each pointing session provides a data set which allows an update of the pointing parameters of the telescope from measurements of the pointing errors of radio sources uniformly distributed over the sky (e.g. Greve et al. 1996). An additional important result of these observations is the determination of the source flux densities. This is done in the following way:

1) The sources are observed with cross-scans in azimuth and elevation direction. The temperature scale of these scans is $T_{\mathrm{A}}^{*}$, which is determined via the standard chopper wheel method (e.g. Mauersberger et al. 1989).

2) Gaussian fits are applied to the individual scans and the temperature scale in each direction is corrected according to the pointing error in the direction perpendicular to it.

3) The corrected antenna temperature is converted into flux density by using the instantaneous conversion factors derived either for Mars or for the HII regions/planetary nebulae W3(OH), K3-50A, NGC 7538 and NGC 7027. In Table 2 we give peak flux densities of these secondary calibrators at the most commonly used frequencies (Reuter et al., in preparation). These fluxes were derived from measurements of Mars assuming disk brightness temperatures of Mars as given by Ulich (1981) and Griffin et al. (1986).

The mean and long-term variation $(1 \sigma)$ of the calibration factors $\left(\mathrm{Jy} \mathrm{K}^{-1}\right)$ at 88,142 and $226 \mathrm{GHz}$ are found to be $5.92 \pm 7 \%, 7.14 \pm 6 \%$ and $10.5 \pm 13 \%$, respectively. We note that these values refer to measurements after March, 
Table 2. Receivers used at the telescope (1993-1994)

\begin{tabular}{llllc}
\hline Rx name & $\nu[\mathrm{GHz}]$ & $\lambda[\mathrm{mm}]$ & $\mathrm{HPBW}\left[^{\prime \prime}\right]$ & $\mathrm{Jy} \mathrm{K}^{-1}$ \\
\hline 3mmS1 & 87.7 & 3.4 & 27 & 5.92 \\
2mmS1 & 142 & 2.1 & 17 & 7.14 \\
230G1 & 225 & 1.3 & 11 & 10.5 \\
\hline
\end{tabular}

Table 3. Secondary calibrator flux densities

\begin{tabular}{lllll}
\hline$\nu[\mathrm{GHz}]$ & W3 $(\mathrm{OH})$ & K3-50A & NGC 7027 & NGC 7538 \\
\hline 87.7 & 3.95 & 6.28 & 4.68 & 2.50 \\
142 & 4.30 & 6.20 & 4.05 & 3.20 \\
225 & 6.31 & 6.91 & 3.68 & 5.06 \\
\hline
\end{tabular}

1994, when the sensitivity of the telescope improved after a re-alignment of the subreflector.

\section{The flux catalogue}

In Table 4 we list the flux densities obtained for the period 1993-1994. This table is published electronically; the data are also available at CDS in Strasbourg. The table is arranged as follows:

Column 1: Observing date,

Column 2: Observing frequency,
Columns 3 and 4: Measured flux density and its error. The quoted error is the root of the quadratic sum of the individual observational error (dependent on weather and pointing offset) and the percentage calibration uncertainty as described in Sect. 2.

\section{Light curves}

In Fig. 1 we show the radio light curves for the time interval 1985-1994 (data before 1993 taken from Papers I-III) of sources for which at least 10 independent flux measurements at $88 \mathrm{GHz}$ were obtained between 1993 and 1994 .

Acknowledgements. It is a pleasure to thank the staff of the 30 $\mathrm{m}$ telescope for the kind support during the observations. W.J. Altenhoff is thanked for his critical reading of the manuscript.

\section{References}

Greve A., Panis J.-F., Thum C., 1996, A\&AS 115, 379

Griffin M.J., Ade P.A.R., Orton G.S., et al., 1986, Icarus 65, 244

Mauersberger R., Guélin M., Martín-Pintado J., et al., 1989, A\&AS 79, 217

Penzias A.A., Burrus C.A., 1973, ARA\&A 11, 51

Steppe H., Salter C.J., Chini R., et al., 1988, A\&AS 75, 317 (Paper I)

Steppe H., Liechti S., Mauersberger R., et al., 1992, A\&AS 96, 441 (Paper II)

Steppe H., Paubert G., Sievers A., et al., 1993, A\&AS 102, 611 (Paper III)

Ulich B.L., 1981, AJ 86, 1619

Wild W., 1995, "The 30m Manual: A Handbook for the $30 \mathrm{~m}$ telescope", IRAM preprint No. 377 

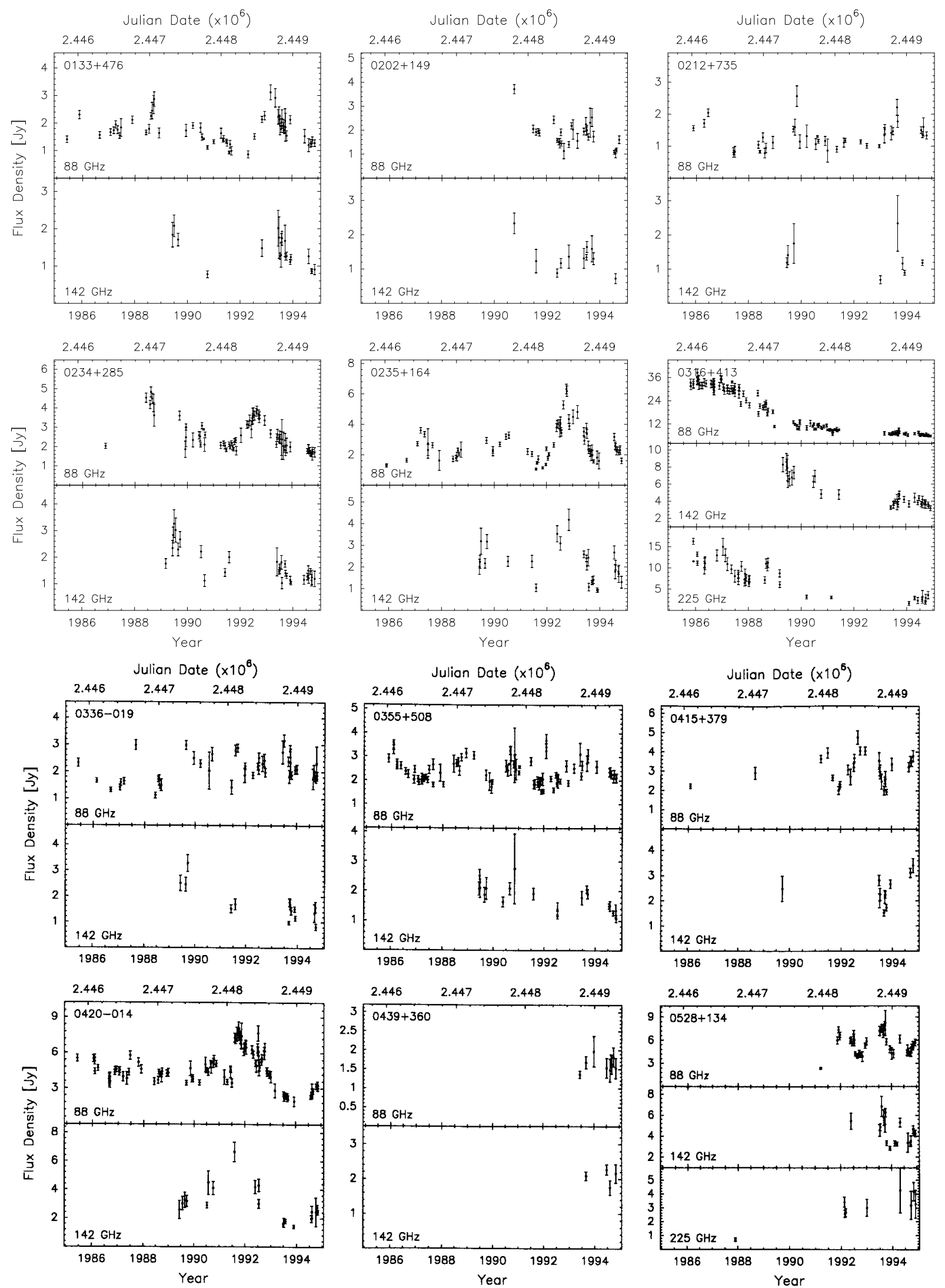

Fig. 1. Light curves for the time interval 1985-1994 of the sources for which at least 10 flux densities at $88 \mathrm{GHz}$ have been obtained between 1993 and 1994 

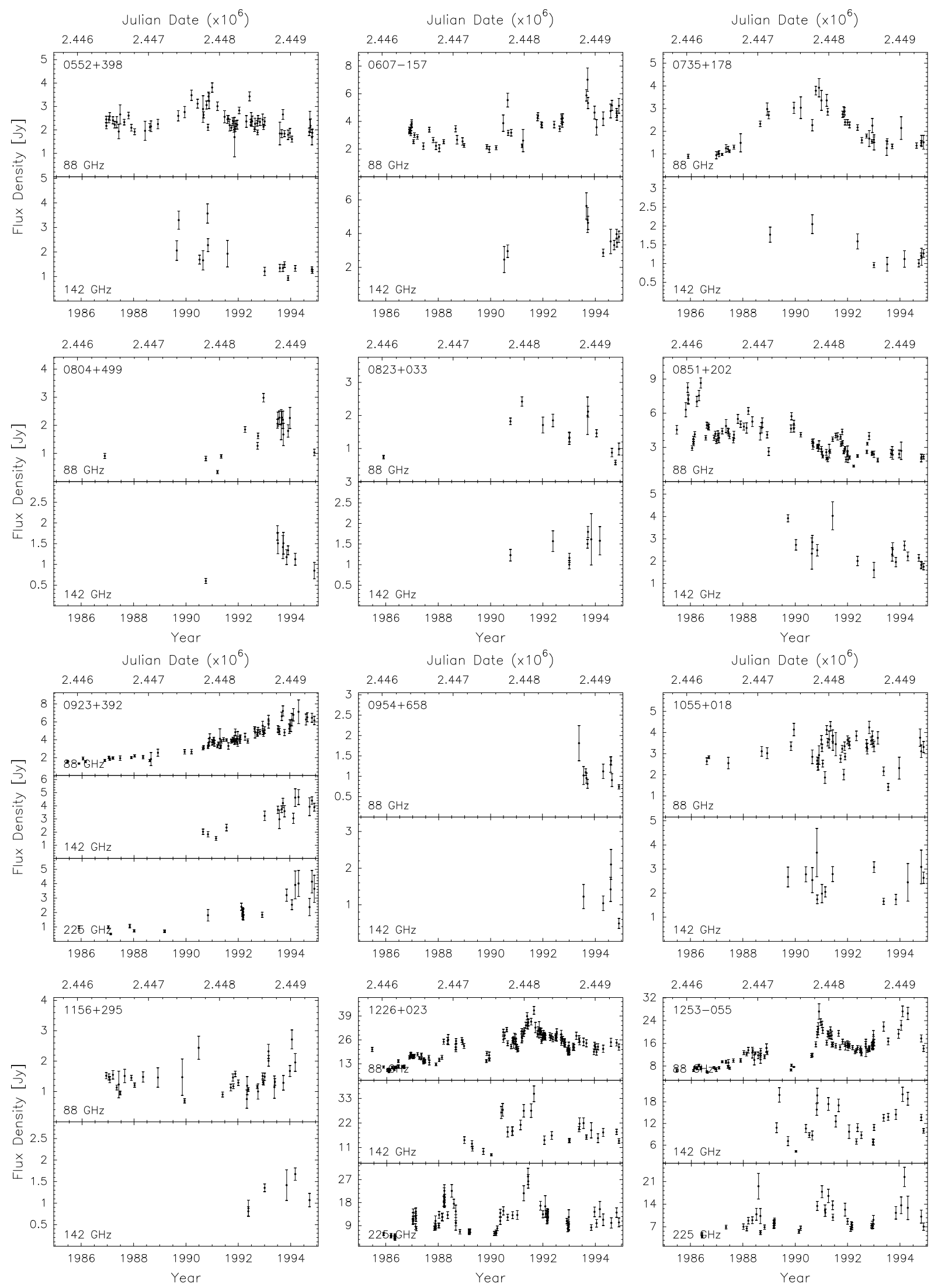

Fig. 1. continued 

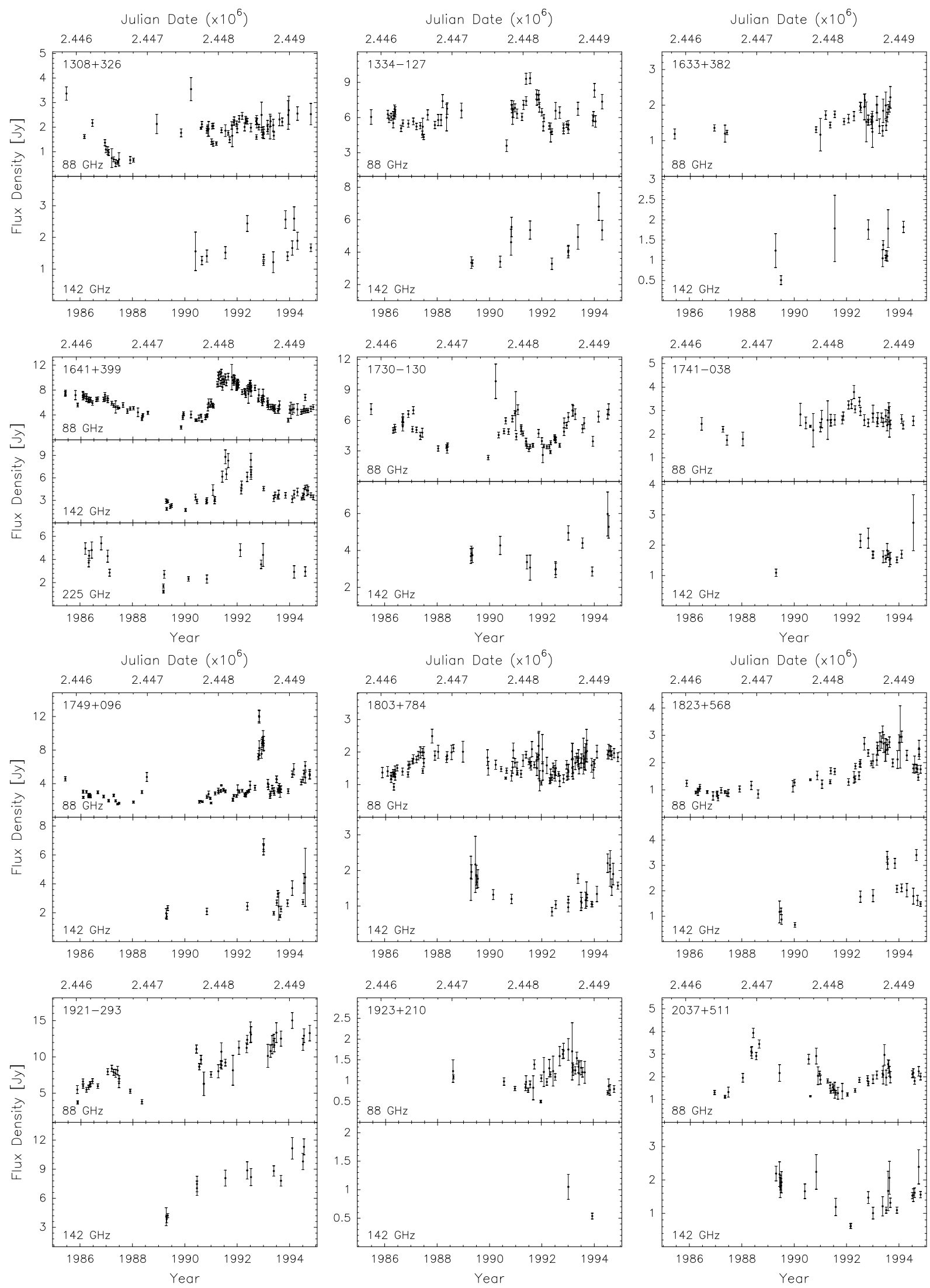

Fig. 1. continued 

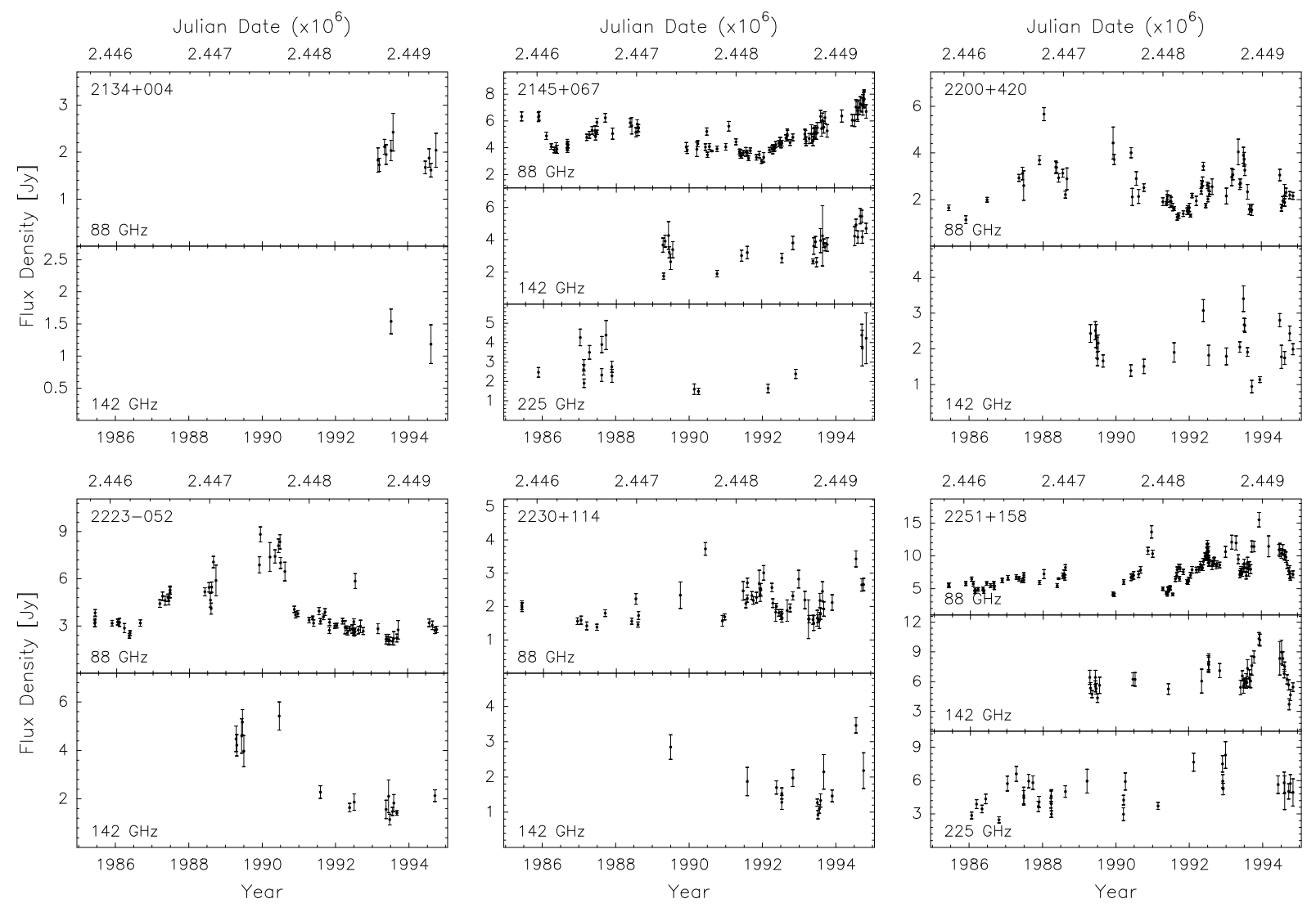

Fig. 1. continued 